Vol. 44 (1991) [155-169]

\title{
FRACTIONS OF THE PERIOD OF THE CONTINUED FRACTION EXPANSION OF QUADRATIC INTEGERS
}

\section{A.J. VAN DER POORTEN}

Dedicated to George Szekeres on his eightieth birthday

The elementary nature and simplicity of the theory of continued fractions is mostly well disguised in the literature. This makes one reluctant to quote sources when making a remark on the subject and seems to necessitate redeveloping the theory $a b$ initio. That had best be done succinctly. That is done here and allows the retrieval of some amusing results on pattern in the period of the continued fraction expansion of quadratic integers.

\section{A fundamental Correspondence}

A continued fraction is an expression of the shape

$$
a_{0}+\frac{1}{a_{1}+\frac{1}{a_{2}+\frac{1}{a_{3}+\frac{1}{\ddots}}}}
$$

which we denote in a space-saving flat notation by

$$
\left[a_{0}, a_{1}, a_{2}, a_{3}, \ldots\right] \text {. }
$$

\section{Proposition 1.}

$$
\left(\begin{array}{cc}
a_{0} & 1 \\
1 & 0
\end{array}\right)\left(\begin{array}{cc}
a_{1} & 1 \\
1 & 0
\end{array}\right) \cdots\left(\begin{array}{cc}
a_{n} & 1 \\
1 & 0
\end{array}\right)=\left(\begin{array}{cc}
p_{n} & p_{n-1} \\
q_{n} & q_{n-1}
\end{array}\right) \text { for } n=0,1,2, \ldots
$$

if and only if

$$
\frac{p_{n}}{q_{n}}=\left[a_{0}, a_{1}, \ldots, a_{n}\right] \text { for } n=0,1,2, \ldots,
$$

Received 1st October, 1990.

Work supported in part by a grant from the Australian Research Council

Copyright Clearance Centre, Inc. Serial-fee code: 0004-9729/91 \$A2.00+0.00. 
Proof: This formal correspondence is easily established by a thoughtful inductive argument on the number of matrices, using

$$
\left[a_{0}, a_{1}, \ldots, a_{n}\right]=a_{0}+\frac{1}{\left[a_{1}, a_{2}, \ldots, a_{n}\right]} .
$$

We will not need to be reminded of the approximation properties of continued fractions for the present discussion. I shall remark, however, that these properties follow readily from taking determinants in the correspondence, thus seeing that

$$
p_{n} q_{n-1}-p_{n-1} q_{n}=(-1)^{n+1} \text { whence } \frac{p_{n}}{q_{n}}=\frac{p_{n-1}}{q_{n-1}}+\frac{(-1)^{n-1}}{q_{n-1} q_{n}} .
$$

If

I write

$$
\begin{aligned}
\gamma & =\left[a_{0}, a_{1}, a_{2}, \ldots\right], \\
\frac{p_{n}}{q_{n}} & =\left[a_{0}, a_{1}, \ldots, a_{n}\right]
\end{aligned}
$$

to denote the convergents $p_{n} / q_{n}$ of $\gamma$. The $a_{n}$ are known as the partial quotients of $\gamma$ and the quantities $\gamma_{n}$ defined by

$$
\gamma=\left[a_{0}, a_{1}, \ldots, a_{n-1}, \gamma_{n}\right]
$$

are called its complete quotients.

Below, almost invariably, but not always - many of the arguments are formal - the partial quotients (other than $a_{0}$ ) will be positive integers. This, the case of admissible partial quotients, and thence of regular continued fractions (we omit the qualifying adjective in the sequel), is our principal concern.

It is an interesting exercise to apply the correspondence and to see that the complete quotients have an expansion given by

$$
-\gamma_{n+1}=\frac{q_{n-1} \gamma-p_{n-1}}{q_{n} \gamma-p_{n}}=\left[0, a_{n}, a_{n-1}, \ldots, a_{1}, a_{0}-\gamma\right]
$$

It will be convenient to note that a periodic expansion

$$
\gamma=\left[a_{0}, \overline{a_{1}, \ldots, a_{r-1}, a_{r}}\right]
$$

is just

$$
\gamma=\left[a_{0}, a_{1}, \ldots, a_{r-1}, a_{r}+\gamma-a_{0}\right]
$$




\section{Continded fractions of Real Quadratic Irrationals}

In the sequel $\boldsymbol{\gamma}$ denotes a real quadratic irrational with conjugate $\boldsymbol{\gamma}^{\prime}$.

Proposition 2. 'Pell's Equation'

$$
\operatorname{Norm}(X-\gamma Y)=(X-\gamma Y)\left(X-\gamma^{\prime} Y\right)=X^{2}-\left(\gamma+\gamma^{\prime}\right) X Y+\gamma \gamma^{\prime} Y^{2}=1
$$

has solutions in nonzero integers $(X, Y)$.

ProOF: There is some integer $d$ so that $d \gamma$ is an algebraic integer; hence we may suppose that $\gamma$ is an integer and afterwards replace $Y$ by $d Y$. Recall that by the box principle one readily sees that there are infinitely many $(x, y) \in \mathbb{Z}^{2}$ so that $|x-\gamma y|<1 / y$, and therefore

$$
\left|(x-\gamma y)\left(x-\gamma^{\prime} y\right)\right|<\frac{\left|x-\gamma^{\prime} y\right|}{y}<\gamma-\gamma^{\prime}+1 .
$$

Thus there is an integer $k$ with $|k|<\gamma-\gamma^{\prime}+1$ so that $\operatorname{Norm}(x-\gamma y)=k$ has infinitely many solutions and, again by the box principle, that equation has pairs of solutions $(x, y)$ and $\left(x^{\prime}, y^{\prime}\right)$ so that $x \equiv x^{\prime}, y \equiv y^{\prime} \bmod k$.

Then

$$
\begin{aligned}
1=\operatorname{Norm}\left(\frac{x-\gamma y}{x^{\prime}-\gamma y^{\prime}}\right) & =\operatorname{Norm}\left(\frac{x x^{\prime}-x y^{\prime}\left(\gamma+\gamma^{\prime}\right)+y y^{\prime} \gamma \gamma^{\prime}}{k}-\frac{x y^{\prime}-x^{\prime} y}{k} \gamma\right) \\
& =\operatorname{Norm}(X-\gamma Y)
\end{aligned}
$$

where the congruences

$$
x x^{\prime}-x y^{\prime}\left(\gamma+\gamma^{\prime}\right)+y y^{\prime} \gamma \gamma^{\prime} \equiv \operatorname{Norm}(x-\gamma y) \text { and } x y^{\prime}-x^{\prime} y \equiv 0 \bmod k
$$

entail that $X$ and $Y$ are integers as required.

Proposition 3. (Continued fraction algorithm) $A$ unimodular matrix

$$
U=\left(\begin{array}{ll}
a & b \\
c & d
\end{array}\right) .
$$

with nonnegative integer entries satisfying $a \geqslant b$ and $a \geqslant c$ has a unique decomposition

$$
U=\left(\begin{array}{cc}
a_{0} & 1 \\
1 & 0
\end{array}\right)\left(\begin{array}{cc}
a_{1} & 1 \\
1 & 0
\end{array}\right) \cdots \cdots\left(\begin{array}{cc}
a_{n} & 1 \\
1 & 0
\end{array}\right)
$$

with positive integers $a_{0}, a_{1}, \ldots, a_{n}$.

Proof: Either $U=I$ (and the product is empty); or, as is easily seen, $a d-b c=$ \pm 1 and $a \geqslant c$ entails that $b \geqslant d$ (dually, $a \geqslant b$ entails $c \geqslant d$ ). Thus the top row dominates the other and since that property persists in the course of applying the Euclidean algorithm to the rows of a unimodular matrix - until the process terminates - we obtain the decomposition as asserted. 
REMARK. Similarly, any unimodular integer matrix has a finite decomposition of the given shape, but, of course, the $a_{h}$ may not all be positive and the decomposition is not unique.

Now let $M$ be the matrix of the Q-linear map 'multiplication by $X-\gamma Y$ ' with respect to the $\mathbb{Q}$-basis $\left\{\gamma^{\prime}, 1\right\}$ of the $Q$-vector space $\mathbb{Q}(\gamma)$. Then

Set

$$
\begin{gathered}
M=\left(\begin{array}{cc}
X & -\gamma \gamma^{\prime} Y \\
Y & X-\left(\gamma+\gamma^{\prime}\right) Y
\end{array}\right) . \\
J=\left(\begin{array}{ll}
0 & 1 \\
1 & 0
\end{array}\right) .
\end{gathered}
$$

It follows from Proposition 3, and the remark, that we may decompose $M J$, obtaining, say,

$$
M J \longleftrightarrow\left[a_{0}, a_{1}, \ldots, a_{r}\right] .
$$

REMARK. It is instructive to confirm that if and only if

$$
a_{0}-\gamma^{\prime}>1>\gamma-a_{0},
$$

we may choose $X$ and $Y$ so that the matrix

$$
\left(\begin{array}{cc}
X-\left(\gamma+\gamma^{\prime}\right) Y & Y \\
-\gamma \gamma^{\prime} Y+a_{0}\left(\gamma+\gamma^{\prime}\right) Y-a_{0} X & X-a_{0} Y
\end{array}\right)
$$

satisfies the conditions of Proposition 3 and there is a unique decomposition corresponding to a continued fraction with admissible partial quotients.

From this point on we suppose that $\gamma=\beta=\sqrt{D}$ or $\gamma=\alpha=(1+\sqrt{D}) / 2$, with $D$ a positive integer $\equiv 1 \bmod 4$, and not a square. Then the conditions of Proposition 3 hold for $M J$.

Because

$$
M J . J\left(\begin{array}{cc}
\gamma+\gamma^{\prime} & 1 \\
1 & 0
\end{array}\right)=\left(\begin{array}{cc}
-\gamma \gamma^{\prime} Y & X \\
X & Y
\end{array}\right)
$$

is symmetric we see (by transposition of the matrix product and uniqueness of the decomposition) that the word

$$
a_{0} a_{1} \ldots a_{r-1} a_{r}^{\prime} \text { with } a_{r}^{\prime}=a_{r}+\left(\gamma+\gamma^{\prime}\right)
$$

is a palindrome [a palindrome is never even; it is a toyota.].

Now consider the periodic continued fraction

$$
\begin{aligned}
\delta & =\left[a_{0}, \overline{a_{1}, \ldots, a_{r-1}, 2 a_{0}-\left(\gamma+\gamma^{\prime}\right)}\right] \\
& =\left[a_{0}, \stackrel{a_{1}, \ldots, a_{r-1}}{\longleftrightarrow}, 2 a_{0}-\left(\gamma+\gamma^{\prime}\right)+\delta-a_{0}\right] .
\end{aligned}
$$


It corresponds to

so

$$
\begin{aligned}
\operatorname{MJ.J}\left(\begin{array}{ll}
\delta & 1 \\
1 & 0
\end{array}\right) & =\left(\begin{array}{cc}
X & -\gamma \gamma^{\prime} Y \\
Y & X-\left(\gamma+\gamma^{\prime}\right) Y
\end{array}\right)\left(\begin{array}{ll}
\delta & 1 \\
1 & 0
\end{array}\right), \\
\delta & =\frac{\delta X-\gamma \gamma^{\prime} Y}{\delta Y+X-\left(\gamma+\gamma^{\prime}\right) Y}
\end{aligned}
$$

or $\delta^{2}-\left(\gamma+\gamma^{\prime}\right) \delta+\gamma \gamma^{\prime}=0$ since $Y \neq 0$. Since $\delta>0$ we have $\delta=\gamma$. Thus

Proposition 4. If $M$ is unimodular then

$$
M J=\left(\begin{array}{cc}
-\gamma \gamma^{\prime} Y & X \\
X-\left(\gamma+\gamma^{\prime}\right) Y & Y
\end{array}\right)=\left(\begin{array}{cc}
a_{0} & 1 \\
1 & 0
\end{array}\right)\left(\begin{array}{cc}
a_{1} & 1 \\
1 & 0
\end{array}\right) \cdots \cdots\left(\begin{array}{cc}
a_{r} & 1 \\
1 & 0
\end{array}\right)
$$

if and only if

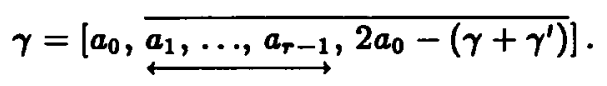

Of course, by the correspondence, $\left[a_{0}, a_{1}, \ldots, a_{r-1}\right]=X / Y$.

COMMENT. If we make no special assumption about the real quadratic irrational $\gamma$ then the same formal argument - omitting the remarks on symmetry - yields

$$
\gamma=\left[a_{0}, \overline{a_{1}, \ldots, a_{r-1}, a_{r}+a_{0}-\left(\gamma+\gamma^{\prime}\right)}\right] .
$$

It can be shown that a periodic continued fraction can always be transformed to a periodic continued fraction with admissible partial quotients; so one obtains an eccentric proof of Lagrange's theorem: Every quadratic irrational has a periodic regular continued fraction expansion. Of course, in general, the transformation to admissible partial quotients may yield a preperiod of different length and may destroy the symmetry of the period. In fact, one obtains a preperiod of length at most 1 if and only if $a_{0}-\gamma^{\prime}>1>\gamma-a_{0}$ and then symmetry as described occurs if and only if $\gamma+\gamma^{\prime} \in \mathbb{Z}$. One obtains a pure symmetric period

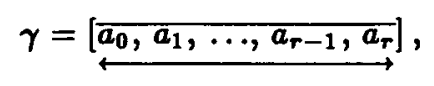

if and only if $\gamma>1$ and $\gamma \gamma^{\prime}=-1$.

\section{HALF THE PERIOD}

We shall synthesise the period of $\gamma$ from half its period and detail the nature of the complete quotients at the centre of the period. 
COMMENT. For $\gamma=\beta=\sqrt{D}$ this material is well-known but in more general cases it seems only implicit in the literature. We have in mind just the additional case $\gamma=\alpha=(1+\sqrt{D}) / 2$ but our remarks remain valid whenever the matrices decompose uniquely to yield admissible partial quotients. In particular, the condition $\gamma+\gamma^{\prime} \in \mathbb{Z}$ is essential.

The following useful lemma plays an important rôle:

Proposition 5. Given $\left[a_{0}, a_{1}, \ldots, a_{h}\right]=p_{h} / q_{h}$, set

$$
M_{h}=\left(\begin{array}{cc}
p_{h} & -\gamma \gamma^{\prime} q_{h} \\
q_{h} & p_{h}-\left(\gamma+\gamma^{\prime}\right) q_{h}
\end{array}\right) .
$$

Then

$$
M_{h} J=\left(\begin{array}{cc}
a_{0} & 1 \\
1 & 0
\end{array}\right)\left(\begin{array}{cc}
a_{1} & 1 \\
1 & 0
\end{array}\right) \cdots \cdots\left(\begin{array}{cc}
a_{h} & 1 \\
1 & 0
\end{array}\right)\left(\begin{array}{cc}
P_{h+1} & 1 \\
Q_{h+1} & 0
\end{array}\right)
$$

where

$$
\frac{\gamma+P_{h+1}}{Q_{h+1}}=\gamma_{h+1}
$$

yields the complete quotient $\gamma_{h+1}$ defined by $\gamma=\left[a_{0}, a_{1}, \ldots, a_{h}, \gamma_{h+1}\right]$.

REMARK. Notice that the assertion defines $Q_{h+1}$ by

$$
\operatorname{Norm}\left(p_{h}-\gamma q_{h}\right)=(-1)^{h+1} Q_{h+1} .
$$

Moreover, it is easy to confirm by induction - in effect this is the inversion in the continued fraction algorithm - that

$$
-\operatorname{Norm}\left(\gamma+P_{h+1}\right)=Q_{h} Q_{h+1} .
$$

Proof: The first allegation of the Proposition is

$$
M_{h} J=\left(\begin{array}{ll}
p_{h} & p_{h-1} \\
q_{h} & q_{h-1}
\end{array}\right)\left(\begin{array}{ll}
P_{h+1} & 1 \\
Q_{h+1} & 0
\end{array}\right)
$$

and that the final matrix in the decomposition is of the alleged shape is now easy to verify. To see the principal claim multiply $M_{h} J$ on the right by

$$
Q_{h+1}^{-1} J\left(\begin{array}{cc}
\gamma & Q_{h+1} \\
1 & 0
\end{array}\right)
$$

and apply the correspondence appropriately: Namely, we obtain

$$
\begin{aligned}
\gamma \longleftrightarrow\left(\begin{array}{cc}
\gamma\left(p_{h}-q_{h} \gamma^{\prime}\right) / Q_{h+1} & p_{h} \\
\left(p_{h}-q_{h} \gamma^{\prime}\right) / Q_{h+1} & q_{h}
\end{array}\right) \\
=\left(\begin{array}{cc}
a_{0} & 1 \\
1 & 0
\end{array}\right)\left(\begin{array}{cc}
a_{1} & 1 \\
1 & 0
\end{array}\right) \cdots \cdots\left(\begin{array}{cc}
a_{h} & 1 \\
1 & 0
\end{array}\right)\left(\begin{array}{cc}
\left(\gamma+P_{h+1}\right) / Q_{h+1} & 1 \\
1 & 0
\end{array}\right) \\
\longleftrightarrow\left[a_{0}, \ldots \ldots, a_{h}, \frac{\gamma+P_{h+1}}{Q_{h+1}}\right] .
\end{aligned}
$$


To compose two initial parts of the period we note that a product $M_{k} M_{k}$ is the matrix of multiplication by

$$
\left(p_{h}-\gamma q_{h}\right)\left(p_{k}-\gamma q_{k}\right)=\left(p_{k} p_{h}-\gamma \gamma^{\prime} q_{k} q_{h}\right)-\gamma\left(p_{k} q_{h}-p_{h} q_{k}-\left(\gamma+\gamma^{\prime}\right) q_{k} q_{h}\right)
$$

and thus, for certain integers $x, y$ the product $M_{h} M_{k} J$ has shape

$$
\left(\begin{array}{cc}
-\gamma \gamma^{\prime} y & x \\
x-\left(\gamma+\gamma^{\prime}\right) y & y
\end{array}\right)
$$

In the sequel it will be useful to set

$$
L=\left(\begin{array}{ll}
1 & 0 \\
1 & 1
\end{array}\right) \text { and } R=\left(\begin{array}{ll}
1 & 1 \\
0 & 1
\end{array}\right)
$$

Later, we will need to notice that

$$
\left(\begin{array}{ll}
a & 1 \\
1 & 0
\end{array}\right)=J L^{a}=R^{a} J
$$

Multiplication on the right by $L^{\gamma+\gamma^{\prime}}$ adds $\gamma+\gamma^{\prime}$ times the second column of the multiplicand to its first column. Thus $M_{k} J L^{\gamma+\gamma^{\prime}}$ is symmetric, which is to say that it equals its transpose $R^{\gamma+\gamma^{\prime}} J^{t} M_{k}=J L^{\gamma+\gamma^{\prime} t} M_{k}$. Hence

$$
\begin{aligned}
& M_{h} M_{k} J L^{\gamma+\gamma^{\prime}}=M_{h} J L^{\gamma+\gamma^{\prime} t} M_{k} \\
& =\left(\begin{array}{cc}
a_{0} & 1 \\
1 & 0
\end{array}\right)\left(\begin{array}{cc}
a_{1} & 1 \\
1 & 0
\end{array}\right) \cdots\left(\begin{array}{cc}
a_{h} & 1 \\
1 & 0
\end{array}\right)\left(\begin{array}{cc}
P_{h+1}+\left(\gamma+\gamma^{\prime}\right) & 1 \\
Q_{h+1} & 0
\end{array}\right)\left(\begin{array}{cc}
1 & 0 \\
P_{k+1} & Q_{k+1}
\end{array}\right) \\
& \times\left(\begin{array}{cc}
a_{k} & 1 \\
1 & 0
\end{array}\right)\left(\begin{array}{cc}
a_{k-1} & 1 \\
1 & 0
\end{array}\right) \cdots\left(\begin{array}{cc}
a_{0} & 1 \\
1 & 0
\end{array}\right) \\
& =\left(\begin{array}{cc}
a_{0} & 1 \\
1 & 0
\end{array}\right)\left(\begin{array}{cc}
a_{1} & 1 \\
1 & 0
\end{array}\right) \cdots\left(\begin{array}{cc}
a_{h} & 1 \\
1 & 0
\end{array}\right)\left(\begin{array}{cc}
P_{h+1}+P_{k+1}+\left(\gamma+\gamma^{\prime}\right) & Q_{k+1} \\
Q_{h+1} & 0
\end{array}\right) \\
& \times\left(\begin{array}{cc}
a_{k} & 1 \\
1 & 0
\end{array}\right)\left(\begin{array}{cc}
a_{k-1} & 1 \\
1 & 0
\end{array}\right) \cdots\left(\begin{array}{cc}
a_{0} & 1 \\
1 & 0
\end{array}\right) \text {. }
\end{aligned}
$$

Now suppose that both

$$
Q_{h+1}=Q_{k+1} \text { and } P_{h+1}+P_{k+1}+\left(\gamma+\gamma^{\prime}\right)=a Q_{h+1} \text {, some integer } a .
$$

Then, and only then, the symmetric matrix $Q_{h+1}^{-1} M_{h} M_{k} J L^{\gamma+\gamma^{\prime}}$ is unimodular and has a decomposition

$$
\left(\begin{array}{cc}
a_{0} & 1 \\
1 & 0
\end{array}\right)\left(\begin{array}{cc}
a_{1} & 1 \\
1 & 0
\end{array}\right) \cdots\left(\begin{array}{cc}
a_{h} & 1 \\
1 & 0
\end{array}\right)\left(\begin{array}{cc}
a & 1 \\
1 & 0
\end{array}\right)\left(\begin{array}{cc}
a_{k} & 1 \\
1 & 0
\end{array}\right)\left(\begin{array}{cc}
a_{k-1} & 1 \\
1 & 0
\end{array}\right) \cdots\left(\begin{array}{cc}
a_{0} & 1 \\
1 & 0
\end{array}\right)
$$


displaying, according to Proposition 4, the periodic continued fraction

$$
\gamma=\left[a_{0}, \overleftrightarrow{a_{1}, \ldots, a_{h}, a, a_{k}, a_{k-1}, \ldots, a_{1}, 2 a_{0}-\left(\gamma+\gamma^{\prime}\right)}\right] .
$$

In particular, the word $a_{1} \ldots a_{k} a a_{k} a_{k-1} \ldots a_{1}$ is a palindrome.

We denote the length $h+k+2$ of the period of $\gamma$ by $l(\gamma)$.

The conditions

$$
Q_{h+1}=Q_{k+1} \text { and } P_{h+1}+P_{k+1}+\left(\gamma+\gamma^{\prime}\right)=a Q_{h+1} \text {, some integer } a \text {, }
$$

amount to $\quad \gamma_{k+1}=\frac{\gamma+P_{h+1}}{Q_{h+1}}=a-\frac{\gamma^{\prime}+P_{k+1}}{Q_{k+1}}=a-\gamma_{k+1}^{\prime}$.

But this is just a step in the continued fraction expansion of $\gamma$ and says both

$$
a=a_{h+1} \text { and } \gamma_{h+2}^{\prime}=\gamma_{k+1}^{\prime} \text {. }
$$

There are two cases according to the parity of $l(\gamma)$ :

If $l(\gamma)$ is odd, which is necessarily $l(\gamma)=2 h+1$, then the symmetric part of the period is of even length $2 h$ so $k=h-1$ and the central steps of the period are

$$
\frac{\gamma+P_{h}}{Q_{h}}=a_{h}-\frac{\gamma^{\prime}+P_{h+1}}{Q_{h}}
$$

and its conjugate equation

$$
\frac{\gamma+P_{h+1}}{Q_{h}}=a_{h}-\frac{\gamma^{\prime}+P_{h}}{Q_{h}} .
$$

Symmetry is occasioned by selfconjugacy of the pair of equations, signalled by

$$
Q_{h+1}=Q_{h}, \text { which is } \operatorname{Norm}\left(\gamma+P_{h+1}\right)=-Q_{h}^{2} \text {. }
$$

If $l(\gamma)$ is even, which is necessarily $l(\gamma)=2 h+2$, then the symmetric part of the period is of odd length $2 h+1$ so $k=h$ and the central step of the period is

$$
\frac{\gamma+P_{h+1}}{Q_{h+1}}=a_{h+1}-\frac{\gamma^{\prime}+P_{h+1}}{Q_{h+1}} .
$$

Symmetry is occasioned by selfconjugacy of the equation, signalled by

$$
P_{h+1}=P_{h+2} \text {. }
$$

PROPOSITION 6. In the case of period of even length, the partial quotient $a_{h+1}$ central to the symmetric portion of the period is odd whenever $\gamma+\gamma^{\prime}$ is odd.

PROOF: We have $2 P_{h+1}+\left(\gamma+\gamma^{\prime}\right)=a_{h+1} Q_{h+1}$. 


\section{A THIRD OF THE PERIOD}

Recall that we have set

$$
\beta=\sqrt{D} \text { and } \alpha=(1+\sqrt{D}) / 2
$$

where $D>0$ is an integer such that $D \neq \square$ and $D \equiv 1 \bmod 4$. We show that a 'nontrivial' solution - that is, one with $y$ odd - to the diophantine equation $x^{2}-D y^{2}= \pm 4$ signals a 'third' of the period of $\beta$ and, in effect, 'corresponds' to the period of $\alpha$ :

If $(p, q) \in \mathbb{Z}^{2}$ and Norm $(p-\alpha q)= \pm 1$ then

$$
p^{2}-\left(\alpha+\alpha^{\prime}\right) p q+\alpha \alpha^{\prime} q^{2}= \pm 1 \text { which is }(2 p-q)^{2}-D q^{2}= \pm 4
$$

Write

$$
x=2 p-q \text { and } y=q \text {. }
$$

Notice that $\beta+\beta^{\prime}=0$ and $\beta \beta^{\prime}=-D$. Accordingly, set

$$
N=\left(\begin{array}{cc}
x & D y \\
y & x
\end{array}\right)
$$

and recall that $\operatorname{det} N= \pm 4$. It follows that $\operatorname{det} N^{3} J=\mp 64$. Indeed, $N^{3} J$ has shape

$$
N^{3} J=8\left(\begin{array}{cc}
D Y & X \\
X & Y
\end{array}\right) \text { with integers } X \text { and } Y \text {. }
$$

This is clear because $(x-\beta y)^{3}=x^{3}+3 D x y^{2}+\beta^{\prime}\left(3 x^{2} y+D y^{3}\right)$ entails

$$
\begin{aligned}
& 8 X=x\left(x^{2}+3 D y^{2}\right)=x\left(\left(x^{2}-D y^{2}\right)+4 D y^{2}\right) \equiv 0 \bmod 8 \\
& 8 Y=y\left(3 x^{2}+D y^{2}\right)=y\left(3\left(x^{2}-D y^{2}\right)+4 D y^{2}\right) \equiv 0 \bmod 8
\end{aligned}
$$

Since $\left(N^{3} J\right) / 8$ is unimodular, by Proposition 4 we have the correspondence

$$
\begin{aligned}
\frac{1}{8} N^{3} J & =\left(\begin{array}{cc}
b_{0} & 1 \\
1 & 0
\end{array}\right)\left(\begin{array}{cc}
b_{1} & 1 \\
1 & 0
\end{array}\right) \cdots\left(\begin{array}{cc}
b_{r} & 1 \\
1 & 0
\end{array}\right) \\
& \longleftrightarrow \beta=\left[b_{0}, \overline{b_{1}, \ldots, b_{r-1}, 2 b_{0}}\right] .
\end{aligned}
$$

Suppose now that $q=y$ is odd. Then, by Proposition 5, for some index $t$ and integer $P_{t+1}$ we have

$$
N J=\left(\begin{array}{cc}
b_{0} & 1 \\
1 & 0
\end{array}\right)\left(\begin{array}{cc}
b_{1} & 1 \\
1 & 0
\end{array}\right) \cdots\left(\begin{array}{cc}
b_{t} & 1 \\
1 & 0
\end{array}\right)\left(\begin{array}{cc}
P_{t+1} & 1 \\
4 & 0
\end{array}\right)
$$


Now, recalling that $N J$ is symmetric so $N J=J^{t} N$, and noting that $J^{2}=I$,

$$
\begin{aligned}
& N^{3} J=N J . J N . J^{t} N= \\
& \left(\begin{array}{cc}
b_{0} & 1 \\
1 & 0
\end{array}\right)\left(\begin{array}{cc}
b_{1} & 1 \\
1 & 0
\end{array}\right) \cdots\left(\begin{array}{cc}
b_{t} & 1 \\
1 & 0
\end{array}\right)\left(\begin{array}{cc}
P_{t+1} & 1 \\
4 & 0
\end{array}\right)\left(\begin{array}{cc}
y & x \\
x & D y
\end{array}\right)\left(\begin{array}{cc}
P_{t+1} & 4 \\
1 & 0
\end{array}\right) \\
& \times\left(\begin{array}{cc}
b_{t} & 1 \\
1 & 0
\end{array}\right)\left(\begin{array}{cc}
b_{t-1} & 1 \\
1 & 0
\end{array}\right) \cdots\left(\begin{array}{cc}
b_{0} & 1 \\
1 & 0
\end{array}\right) \\
& =\left(\begin{array}{cc}
b_{0} & 1 \\
1 & 0
\end{array}\right)\left(\begin{array}{cc}
b_{1} & 1 \\
1 & 0
\end{array}\right) \cdots\left(\begin{array}{cc}
b_{t} & 1 \\
1 & 0
\end{array}\right) \\
& \times\left(\begin{array}{cc}
\left(D-P_{t+1}^{2}\right) y+2 P_{t+1}\left(x+P_{t+1} y\right) & 4\left(x+P_{t+1} y\right) \\
4\left(x+P_{t+1} y\right) & 16 y
\end{array}\right) \\
& \times\left(\begin{array}{cc}
b_{t} & 1 \\
1 & 0
\end{array}\right)\left(\begin{array}{cc}
b_{t-1} & 1 \\
1 & 0
\end{array}\right) \cdots\left(\begin{array}{cc}
b_{0} & 1 \\
1 & 0
\end{array}\right) .
\end{aligned}
$$

By previous remarks we know that each element of the central matrix is divisible by 8 . We thus see that the central third of the period of $\beta=\sqrt{D}$ is given by

$$
\frac{x+P_{t+1} y}{4 y}=\left[b_{t+1}, b_{t+2}, \cdots, b_{r-t-2}\right] \text {. }
$$

One adds $P_{t+1}$ to $x / y=\left[b_{0}, \ldots, b_{t}\right]$, divides by 4 (see my notes [4]) and recalls that by symmetry $b_{r-t-1}=b_{t+1}$.

All this becomes trivial if $y$ is even. For then $x$ is also even, $N J$ is just $2 M J$, with $M J$ corresponding to a period of $\beta$. So $\left(N^{3} J\right) / 8$ corresponds to a period of $\beta$ comprising three times the given period.

If $y$ is odd then so is $x$. The equation $x^{2}-D y^{2}= \pm 4$ is

$$
\left(\frac{x+y}{2}\right)^{2}-\frac{x+y}{2} y-\frac{D-1}{4} y^{2}= \pm 1
$$

and displays the shorter period of $\alpha=(1+\sqrt{D}) / 2$ relative to that of $\beta=\sqrt{D}$.

I now set

$$
A=\left(\begin{array}{ll}
2 & 0 \\
0 & 1
\end{array}\right) \text { and } A^{\prime}=\left(\begin{array}{ll}
1 & 0 \\
0 & 2
\end{array}\right)
$$

The relationship between the respective periods of $\alpha$ and of $\beta$ can then be summarised by noting that, whilst the unimodular matrix

$$
\frac{1}{8} N^{3}=\left(\begin{array}{cc}
X & D Y \\
Y & X
\end{array}\right)=\left(\begin{array}{cc}
X & -\beta \beta^{\prime} Y \\
Y & X-\left(\beta+\beta^{\prime}\right) Y
\end{array}\right)
$$

yields the period of $\beta=\sqrt{D}$, the unimodular matrix

$$
A^{-1} R N R^{-1} A^{\prime-1}=\left(\begin{array}{cc}
\frac{1}{2}(x+y) & -\frac{1}{4}(D-1) y \\
y & \frac{1}{2}(x+y)-y
\end{array}\right)=\left(\begin{array}{cc}
p & -\alpha \alpha^{\prime} q \\
q & p-\left(\alpha+\alpha^{\prime}\right) q
\end{array}\right)
$$

provides the period of $\alpha=(1+\sqrt{D}) / 2$. 


\section{A SIXTH OF THE PERIOD}

I prove a consequence of the well-known identity $2 \times 3=3 \times 2$. The principal result is an ingenious observation of Kaplan and Williams [1] giving a criterion for the equation $x^{2}-D y^{2}=-4$ to have a solution in odd integers $x, y$ given that $X^{2}-D Y^{2}=-1$ has a solution in integers $X, Y$.

THEOREM. Given a quadratic irrational $\gamma$, let $l(\gamma)$ denote the length of the period of its continued fraction expansion. Let $D>0$ be a nonsquare integer $\equiv 1(\bmod 4)$. Then

$$
l(\sqrt{D}) \equiv l\left(\frac{1}{2}(1+\sqrt{D})\right)(\bmod 4)
$$

unless the equation $X^{2}-D Y^{2}=-1$ has a solution in integers $X, Y$ but the equation $x^{2}-D y^{2}=-4$ has no solution in odd integers $x, y$.

Proof: As at Section 3, write

$$
M_{k} J=\left(\begin{array}{cc}
-\frac{1}{4}(1-D) q_{k} & p_{k} \\
p_{k}-q_{k} & q_{k}
\end{array}\right)=\left(\begin{array}{cc}
a_{0} & 1 \\
1 & 0
\end{array}\right)\left(\begin{array}{cc}
a_{1} & 1 \\
1 & 0
\end{array}\right) \cdots\left(\begin{array}{cc}
a_{k} & 1 \\
1 & 0
\end{array}\right)\left(\begin{array}{cc}
P_{k+1} & 1 \\
Q_{k+1} & 0
\end{array}\right)
$$

for $k=0,1, \ldots$ and set

$$
T_{k}=R^{-1} A M_{k} A^{\prime} R=\left(\begin{array}{cc}
2 p_{k}-q_{k} & D q_{k} \\
q_{k} & 2 p_{k}-q_{k}
\end{array}\right) .
$$

In the sequel it will be useful to recall the following transition formulae:

$$
\begin{aligned}
A R & =R^{2} A & A^{\prime} L & =L^{2} A^{\prime} \\
A L^{2} & =L A & A^{\prime} R^{2} & =R A^{\prime} \\
A L R & =R L A^{\prime} & A^{\prime} R L & =L R A .
\end{aligned}
$$

Suppose that $\alpha$ has period of odd length $l(\alpha)=2 h+1$. Then

$$
\begin{aligned}
T_{h} T_{h-1} J=T_{h} J^{t} T_{h-1} & =R^{-1} A M_{h} A^{\prime} R . J . L A^{\prime} M_{h-1} A L^{-1} \\
& =2 R^{-1} A M_{h} J L^{t} M_{h-1} A L^{-1}=2 Q_{h+1} R^{-1} A M_{2 h} J L A L^{-1} \\
& =2 Q_{h+1} R^{-1} A M_{2 h} A^{\prime} R J=2 Q_{h+1}\left(\begin{array}{cc}
D y & x \\
x & y
\end{array}\right) \\
& =2 Q_{h+1} N J .
\end{aligned}
$$

Here we use the easily established identities $A^{\prime} R . J . L A^{\prime}=2 J L$ and $J L A L^{-1}=A^{\prime} R J$. Since $M_{h} M_{h-1} J$ produces $2 h+2$ partial quotients of $\alpha$, the matrix $T_{h} T_{h-1} J=N J$ 
produces a unimodular matrix $U_{2 h}$, say, corresponding to $2 h+1(\bmod 2)$ partial quotients of $\beta$ and a final factor of the shape

$$
Q_{h+1}\left(\begin{array}{cc}
P & 1 \\
4 & 0
\end{array}\right)
$$

and it displays a third of a period of $\beta$ in the sense described at Section 4. Namely, $N^{3} J$ is 8 times a unimodular matrix corresponding to a period of $\beta$. So much for $3 \times 2$.

Thus, a period of $\beta$ corresponds to

$$
\begin{aligned}
8^{-1} N^{3} J=N . N . N J & =N . N . J^{t} N \\
& =\left(8 Q_{h+1}\right)^{-1} N . R^{-1} A M_{h} J L^{t} M_{h-1} A L^{-1} \cdot{ }^{t} N \\
& =\left(8 Q_{h+1}\right)^{-1} N J . J R^{-1} A . M_{h} J L^{t} M_{h-1} \cdot A L^{-1} J . J^{t} N .
\end{aligned}
$$

But we have just been reminded that the central matrices yield

$$
M_{h} . J L .{ }^{t} M_{h-1}=Q_{h+1} M_{h}{ }^{t} M_{h} \text {. }
$$

Hence the period of $\beta$ corresponds to

$$
8^{-1} N R^{-1} A M_{h} \cdot{ }^{t} M_{h} A L^{-1}{ }^{t} N
$$

and is neatly displayed as its apparent halves; this is $2 \times 3$.

We now study the half period: We have

$$
\begin{aligned}
N J . J R^{-1} A M_{h} & =U_{2 h}\left(\begin{array}{cc}
P & 1 \\
4 & 0
\end{array}\right) \cdot J R^{-1} A M_{h} \\
& =U_{2 h}\left(\begin{array}{cc}
2 & P-1 \\
0 & 4
\end{array}\right) M_{h}=2 U_{2 h}\left(\begin{array}{cc}
1 & \frac{1}{2}(P-1) \\
0 & 2
\end{array}\right) M_{h},
\end{aligned}
$$

noting that $P-1$ is even because, as remarked at Section $3,4 \mid\left(D-P^{2}\right)$.

It remains to implicitly apply the transition formulae and to write

$$
U_{2 h}\left(\begin{array}{cc}
1 & \frac{1}{2}(P-1) \\
0 & 2
\end{array}\right) M_{h}
$$

as a product of matrices, $W$ say, corresponding to $3 h+2(\bmod 2)$ partial quotients and a matrix of determinant 2. By the transition formulae the possibilities for that last matrix are $A, A^{\prime}, A L$ or $A^{\prime} R$.

But neither $A$ nor $A^{\prime}$ is possible. If, for example, that matrix were $A$ then we would have the period of $\beta$ corresponding to

$$
2^{-1} W A \cdot A^{t} W
$$


whereas $A A$ is not divisible by 2 . Dually, neither is $A^{\prime} A^{\prime}$. However

$$
A L . R A=R L A^{\prime} A=2 R L=2\left(\begin{array}{ll}
1 & 1 \\
1 & 0
\end{array}\right)\left(\begin{array}{ll}
1 & 1 \\
1 & 0
\end{array}\right)
$$

and dually $A^{\prime} R . L A^{\prime}=L R A A^{\prime}=2 L R$ yields the same matrices.

Thus the given period of $\beta$ corresponds to

$$
W\left(\begin{array}{ll}
1 & 1 \\
1 & 0
\end{array}\right) \cdot\left(\begin{array}{ll}
1 & 1 \\
1 & 0
\end{array}\right)^{t} W
$$

This period has odd length $2 p+1$, say, with the last equation revealing that

$$
p+1 \equiv 3 h+3 \quad(\bmod 2) ; \text { thus } 2 p+1 \equiv 2 h+1 \quad(\bmod 4)
$$

If $x^{2}-D y^{2}=-4$ has a nontrivial solution, that is with $y$ odd, then we have obtained the primitive period of $\beta$ and its length $l(\beta)$ satisfies $l(\beta)=2 p+1 \equiv 2 h+1=$ $l(\alpha)(\bmod 4)$.

However, if $x^{2}-D y^{2}= \pm 4$ entails $y$ even then we have been dealing with three times a period and have shown that $3 l(\beta)=2 p+1$. In this case we obtain $l(\beta) \equiv$ $2 p-1 \equiv(2 h+1)-2(\bmod 4)$.

If $\alpha$ has only periods of even length then we may apply a similar argument with $M_{h}$ replacing $M_{h-1}$. Other than for

$$
M_{h} . J L \cdot{ }^{t} M_{h}=Q_{h+1} M_{h} \cdot\left(\begin{array}{cc}
a_{h+1} & 1 \\
1 & 0
\end{array}\right) \cdot{ }^{t} M_{h}
$$

the argument proceeds as above until we come to determine which of the products

$$
A L\left(\begin{array}{cc}
a_{h+1} & 1 \\
1 & 0
\end{array}\right) R A=J A^{\prime} R L^{a_{h+1}} R A
$$

and so forth, is divisible by 2 . A computation shows that the only admissible configu- 
ration is:

$$
A\left(\begin{array}{cc}
a_{h+1} & 1 \\
1 & 0
\end{array}\right) A=J A^{\prime} L^{a_{h+1}} A=J L^{2 a_{h+1}} A^{\prime} A=2\left(\begin{array}{cc}
2 a_{h+1} & 1 \\
1 & 0
\end{array}\right)
$$

Of course we do have

$$
\begin{gathered}
A^{\prime}\left(\begin{array}{cc}
a_{h+1} & 1 \\
1 & 0
\end{array}\right) A^{\prime}=J A L^{a_{h+1}} A^{\prime}=J L^{\frac{1}{2} a_{h+1}} A A^{\prime}=2\left(\begin{array}{cc}
\frac{1}{2} a_{h+1} & 1 \\
1 & 0
\end{array}\right) ; \\
A^{\prime} R\left(\begin{array}{cc}
a_{h+1} & 1 \\
1 & 0
\end{array}\right) L A^{\prime}=J A L^{a_{h+1}+2} A^{\prime}=J L^{\frac{1}{2} a_{h+1}+1} A A^{\prime}=2\left(\begin{array}{cc}
\frac{1}{2} a_{h+1}+1 & 1 \\
1 & 0
\end{array}\right) ;
\end{gathered}
$$

and

$$
\begin{gathered}
A L\left(\begin{array}{cc}
a_{h+1} & 1 \\
1 & 0
\end{array}\right) R A=J A^{\prime} R L^{a_{h+1}} R A=J L R A L^{a_{h+1}-2} L R A \\
=J L R L^{\frac{1}{2} a_{h+1}-1} A L R A=J L R L^{\frac{1}{3} a_{h+1}-1} R L A^{\prime} A \\
=2\left(\begin{array}{ll}
1 & 1 \\
1 & 0
\end{array}\right)\left(\begin{array}{ll}
1 & 1 \\
1 & 0
\end{array}\right)\left(\begin{array}{cc}
\frac{1}{2} a_{h+1}-1 & 1 \\
1 & 0
\end{array}\right)\left(\begin{array}{ll}
1 & 1 \\
1 & 0
\end{array}\right)\left(\begin{array}{ll}
1 & 1 \\
1 & 0
\end{array}\right) .
\end{gathered}
$$

Each of these last three cases is applicable provided that $a_{h+1}$ is even. But by Proposition 6 the central partial quotient is odd. Thus the apparent half period of $\beta$, which is of length $3 h+3(\bmod 2)$, is not augmented by any partial quotients. We have

$$
p+1 \equiv 3 h+3(\bmod 4) \text { which is } l(\beta)=2 p+2 \equiv 2 h+2=l(\alpha)(\bmod 4) .
$$

Moreover, it is not now relevant that $x^{2}-D y^{2}= \pm 4$ have a solution with $y$ odd. For if $l(\beta)$ is even then $3 l(\beta) \equiv l(\beta)(\bmod 4)$.

REMARK. The present results are remarkable because one expects to be able to report at most on the parity, but not on behaviour ( $\bmod 4)$ of periods of continued fractions. One succeeds here by using symmetry and working with the parity of the half periods.

Confession. It is easy to be sloppy and to presume that it suffices to count the parity of the apparent half-period, without bothering to perform the transitions and to compute their consequence. To obtain the Kaplan and Williams [1] result one has to miscount the parity. I found that no obstacle until a referee detected that my miscounting had led me to an additional observation that was palpably false. I no longer believe that the present argument is all that much simpler than that of [1].

\section{Comments and ACKNowledgments}

The bible of the subject is Perron's Kettenbrüche [2]. Its language makes it inaccessible to many. 
There is little in my remarks that is not well-known, but much of it is not widely known. I hope to have shown that all of it can be known readily from first principles. The reader will find it of interest to compare the proof in [1] of the Theorem with that given here. I am indebted to Hugh Williams for challenging me to discover the results of Sections 4-5 armed with no more than an ability to multiply $2 \times 2$ matrices. Rick Mollin showed me the symmetry of a toyota. Harold Stark's treatment [3] of continued fractions influenced my thinking. I am grateful to P. Majstrenko for some helpful questions about details in a draft of this note. Ross Talent has helped me to avoid some of the sloppiness to which I am prone.

It is natural to ask whether one may usefully find yet smaller natural fractions of the period. I believe that the fractional parts shown are the only ones detectable from the complete quotients, thus from the $P_{n}$ and $Q_{n}$, alone. If one knows the convergents, that is: the $p_{n}$ and $q_{n}$, a little more can be said; I make some laboured remarks on that subject in [4]. I do not think that those results are of computational or algebraic significance.

This paper is the refined and revised text of a talk given at the 2nd Conference of the Canadian Number Theory Association, Vancouver, 1989. The author's attendance at that meeting was assisted by the University of Calgary.

\section{REFERENCES}

[1] Pierre Kaplan and Kenneth S. Williams, 'Pell's equation $X^{2}-m Y^{2}=-1,-4$ and continued fractions", $J$. Number Theory 23 (1986), 169-182.

[2] Oskar Perron, Die Lehre von den Kettenbrüchen, Chelsea reprint of 1929 edition.

[3] H. M. Stark, An introduction to number theory (MIT Press, 1978).

[4] A. J. van der Poorten, 'An introduction to continued fractions', in Diophantine Analysis, Editors J.H. Loxton and A. J. van der Poorten, pp. 99-138 (Cambridge University Press, 1986).

School of Mathematics, Physics,

Computing and Electronics

Macquarie University NSW 2109

Australia 